\title{
The formation of young B/PS bulges in edge-on barred galaxies
}

\author{
H. Wozniak ${ }^{1}$ and L. Michel-Dansac ${ }^{2}$ \\ ${ }^{1}$ Observatory of Lyon, F-69561 Saint-Genis-Laval cedex, France \\ email: herve.wozniak@obs.univ-lyon1.fr \\ ${ }^{2}$ IATE, Observatorio astronomico, X5000BGR Cordoba, Argentina \\ email: leo@oac.uncor.edu
}

\begin{abstract}
We report about the fact that the stellar population that is born in the gas inflowing towards the central regions can be vertically unstable leading to a B/PS feature remarkably bluer that the surrounding bulge. Using new chemodynamical simulations we show that this young population does not remain as flat as the gaseous nuclear disc and buckles out of the plane to form a new boxy bulge. We show that such a young B/PS bulge can be detected in colour maps.
\end{abstract}

Keywords. galaxies: bulges, galaxies: evolution, galaxies: formation, galaxies: nuclei, galaxies: stellar content, galaxies: structure, methods: n-body simulations

In the generic case of pure $\mathrm{N}$-body simulations, whenever a disc galaxy forms a bar, a B/PS bulge develops in a few dynamical times. In the case of chemodynamical simulations, with gas and star formation/feedback recipes (cf. Wozniak \& Michel-Dansac 2007, Michel-Dansac \& Wozniak 2008 for full details), the B/PS growing process is different and more complicated due to the presence of a young stellar population that is born in the disc. Most of this young population lies in a razor-like central disc during the first 450 Myr, obviously because of the small vertical scaleheight of the initial gas distribution. Since a razor-thin disc is highly unstable the most central part of the disc starts to thicken out the equatorial plane. In roughly a bar rotation period, the vertical distribution gets symmetrically peanut shaped over the central $2 \mathrm{kpc}$, while the young bar is approximately $8 \mathrm{kpc}$ long (cf. Fig $1, t=600 \mathrm{Myr}$ ). At this time, the total mass of the central disc being still low, the thickening process has no clear detectable effect on the whole mass distribution. Then, the old population also starts to buckle out leading to a larger B/PS bulge (cf Fig. 1, $t=1500 \mathrm{Myr}$ ). Both populations being fully mixed, the two components can only be splitted in numerical simulations.

To observationally detect the young stellar component of a B/PS bulge we have to rely on stellar population tracers, e.g. B-V maps. We thus obtained mock $\mathrm{B}-\mathrm{V}$ maps calibrating our simulations. The full process is described by Michel-Dansac \& Wozniak (2004). We assume that the initial population starts with an age of 10.4 Gyr and has a solar metallicity at $z=0$.

Due to the colour contrast between both stellar populations, the young B/PS structure is clearly visible (Fig. 2). The dust could however hampered such a detection (Fig. 2, right panels). At $t=600 \mathrm{Myr}$, since the new stellar disc is young and star formation still active, the dust amount should be likely large. Afterward, the peanut-shape widens out as the young disc evolves. The thick part of the disc doubles its radial size in less than 1 Gyr. The vertical scaleheight also increases with time leading to a well-developed peanut-shaped bulge for $t>1500$ Myr. 

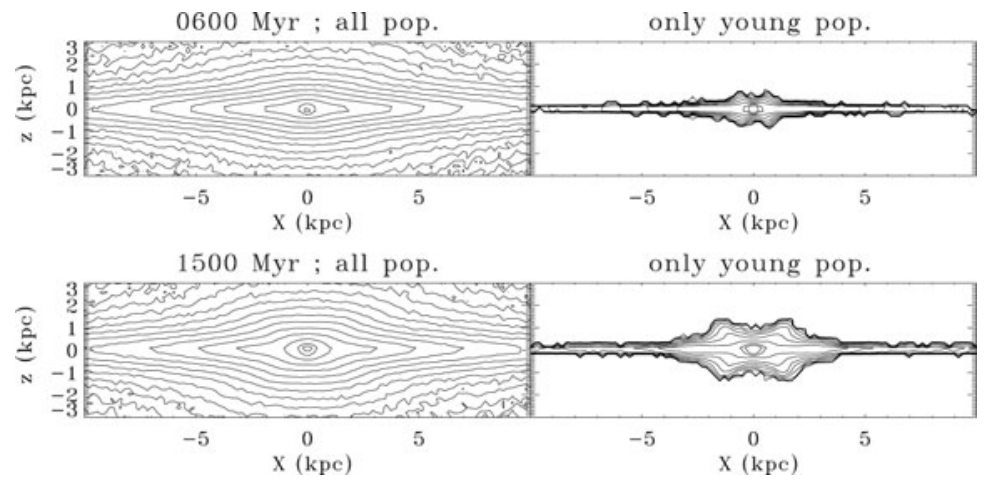

Figure 1. Mass distribution (in log units) of the central $10 \times 5 \mathrm{kpc}$ seen edge-on for $\mathrm{T}=600$ (top panel) and $1500 \mathrm{Myr}$ (bottom). Left panel: all stars (old and young populations) are plotted. Right panel: only the young population has been plotted.
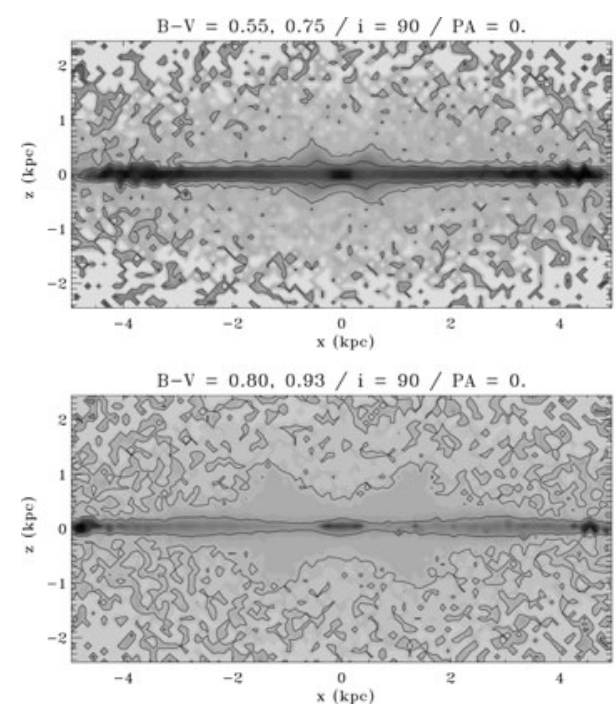
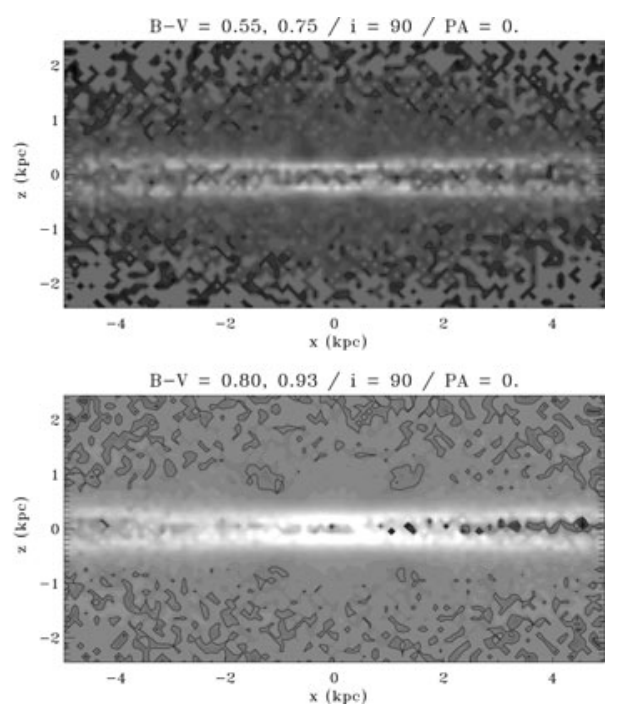

Figure 2. B-V colour maps without (left) and with (right) a dust component for the same fields and times. Bluest region are coded in black. The two isocontours have been chosen as to enhance the boxy feature and are quoted at top of each frame.

The young B/PS structure, being now much more extended both in the radial and vertical direction, is likely detectable even in the presence of dust. Indeed, the height of the $\mathrm{B} / \mathrm{PS}$ structure is greater that the dust disc scaleheight.

Since the early studies of B/PS bulges, there were many evidences that galaxies hosting such a feature are edge-on barred disc galaxies, and that the B/PS bulges themselves represent the thickest parts of the bars. But stellar populations of B/PS bulges (and their colours) have been rarely studied. Looking for young, blue and small scaleheight B/PS bulge, likely inside older, redder and larger bulge, deserves dedicated surveys.

\section{References}

Michel-Dansac L. \& Wozniak H. 2004, A\&A, 421, 863

Michel-Dansac L. \& Wozniak H. 2008, A\&AA, submitted

Wozniak H. \& Michel-Dansac L. 2007, MNRAS, submitted 


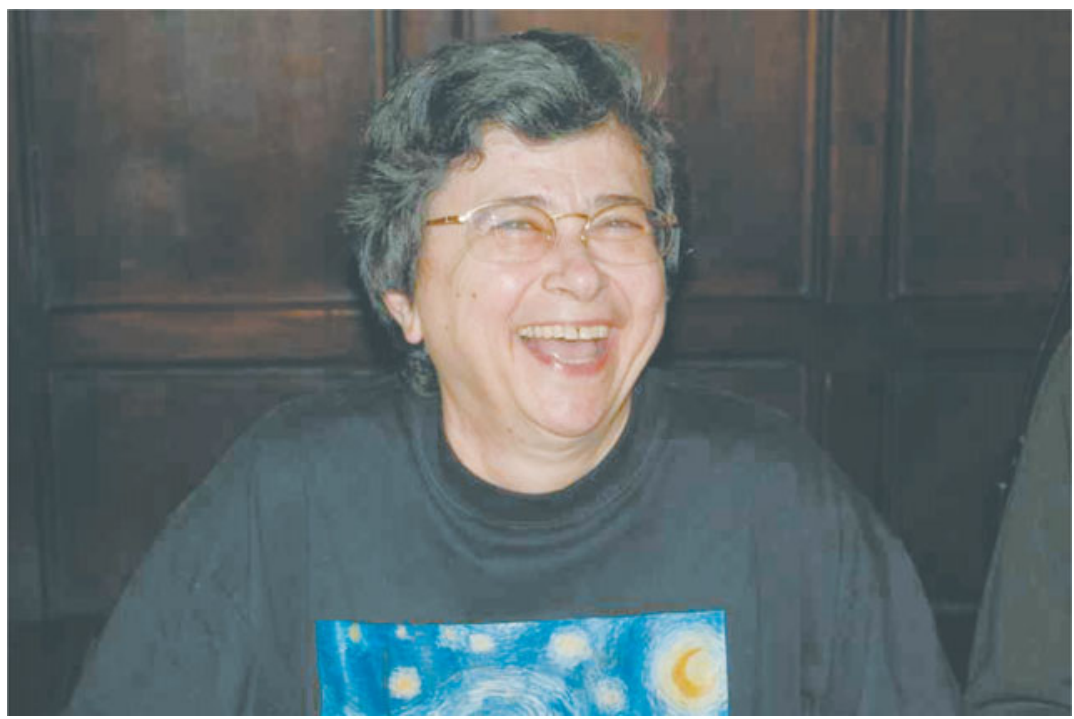

Figure 1. SOC member Lia Athanassoula having a good laugh.

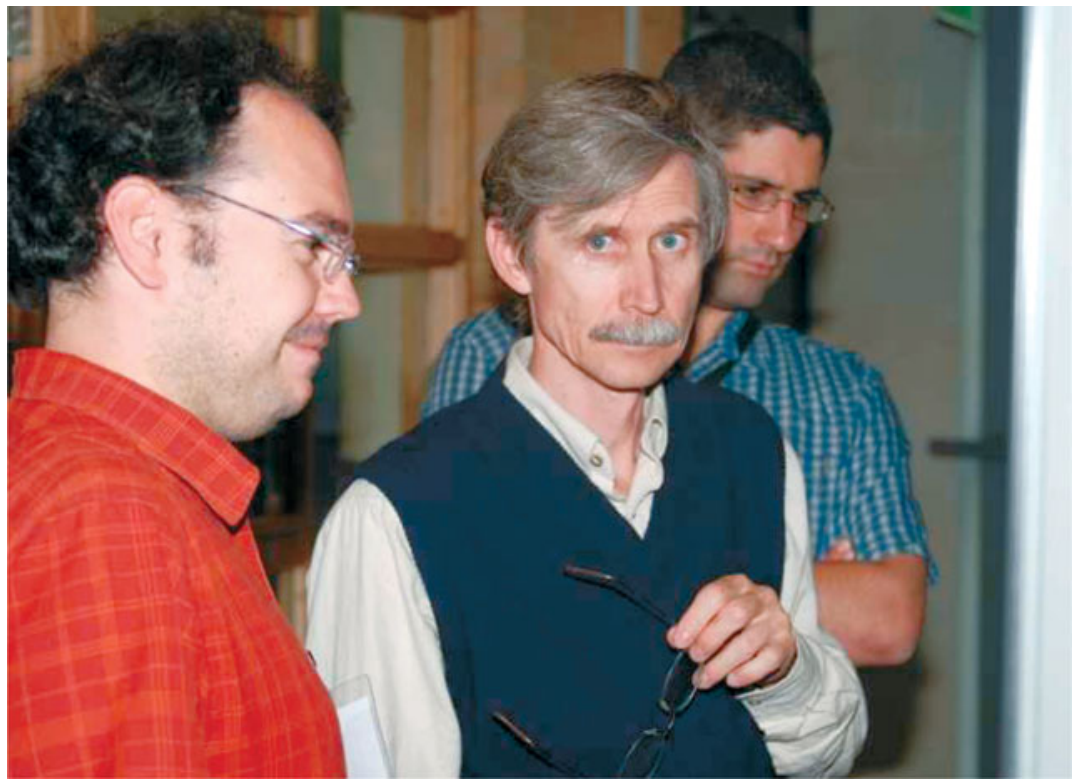

Figure 2. Daniel Ceverino and Anatoly Klypin discussing during the symposium. Elad Zinger is in the background. 


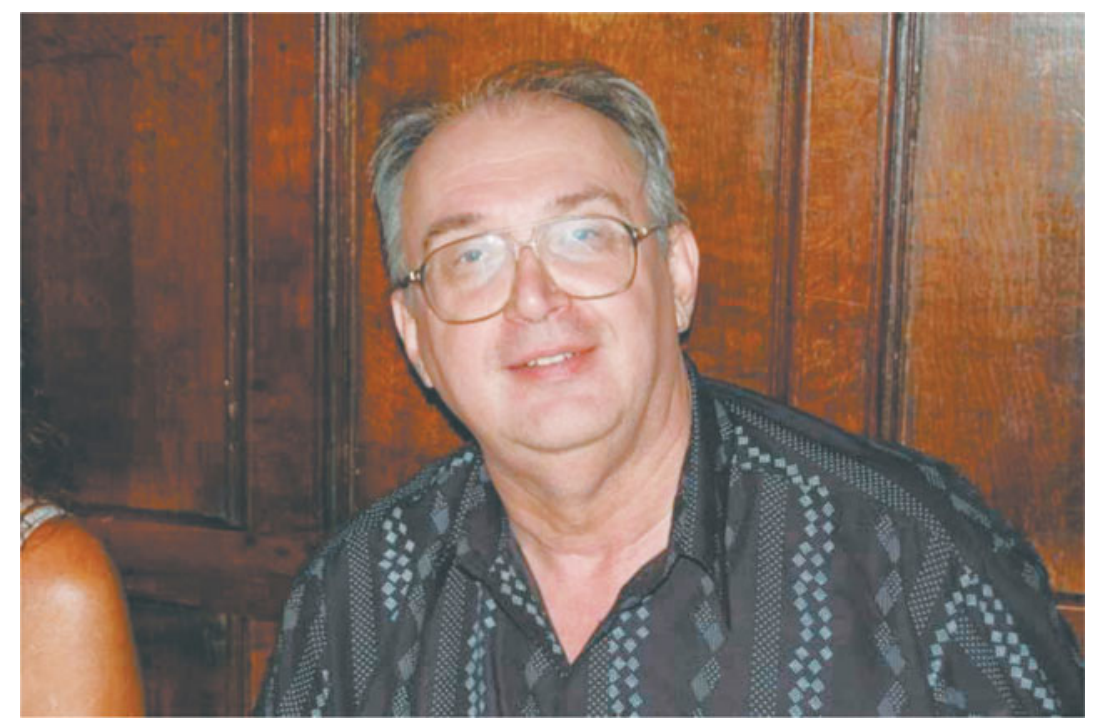

Figure 3. John Kormendy during the symposium dinner.

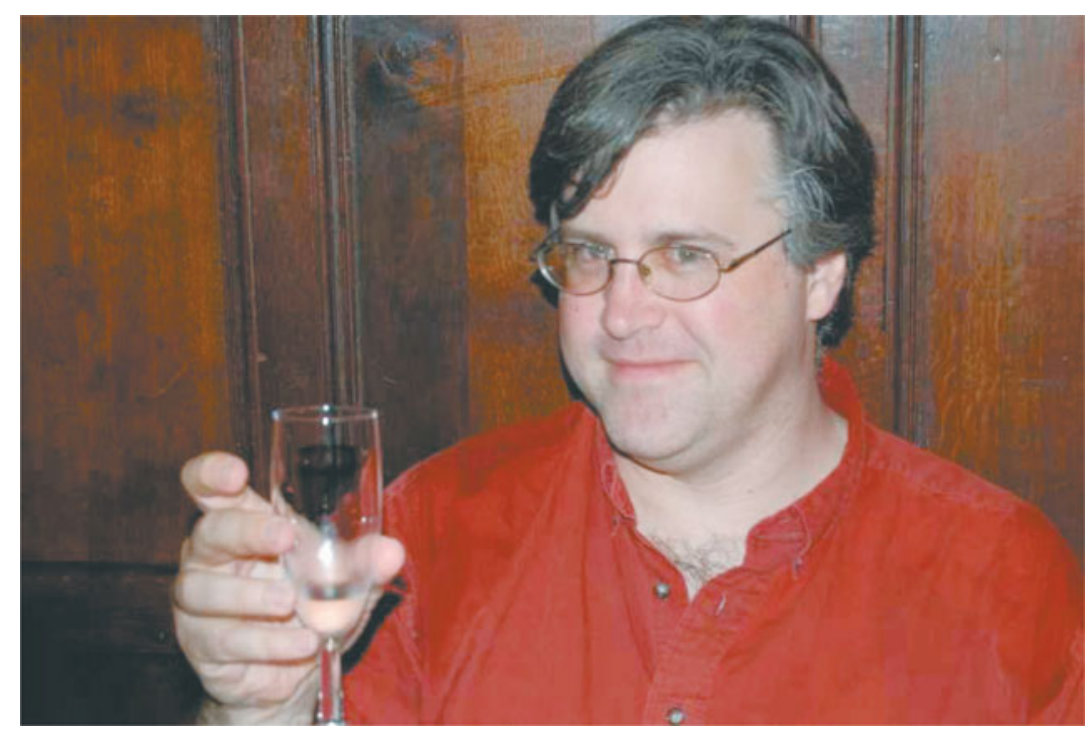

Figure 4. Peter Erwin during the symposium dinner. 


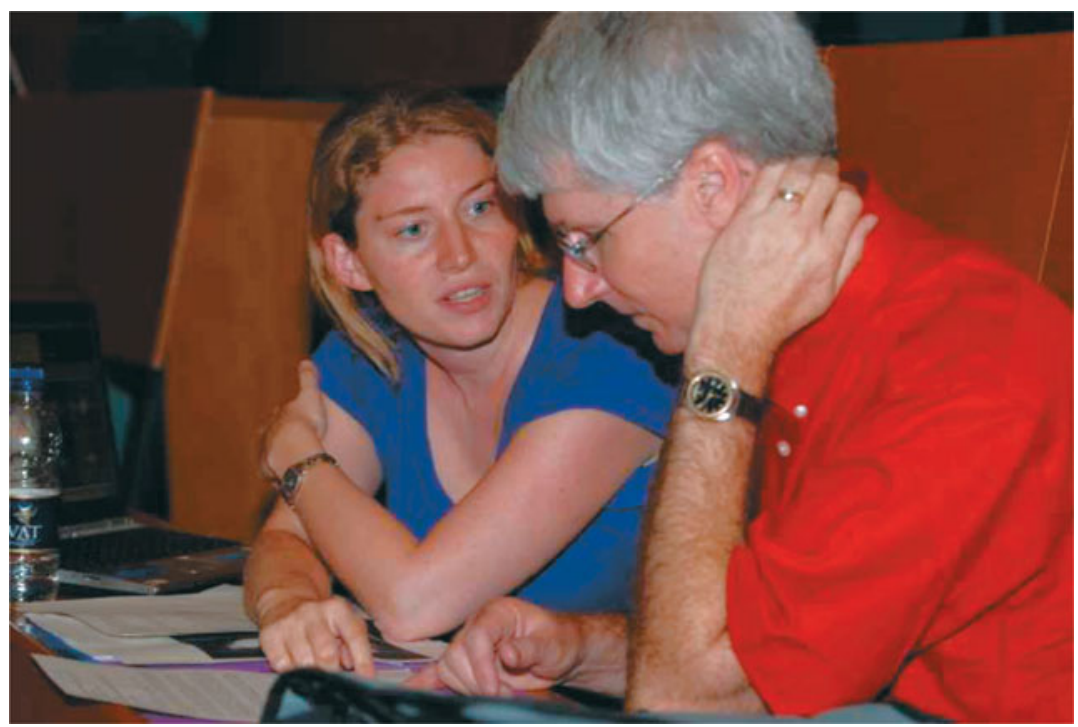

Figure 5. Inma Martinez-Valpuesta and Ronald Buta discussing during a coffee break.

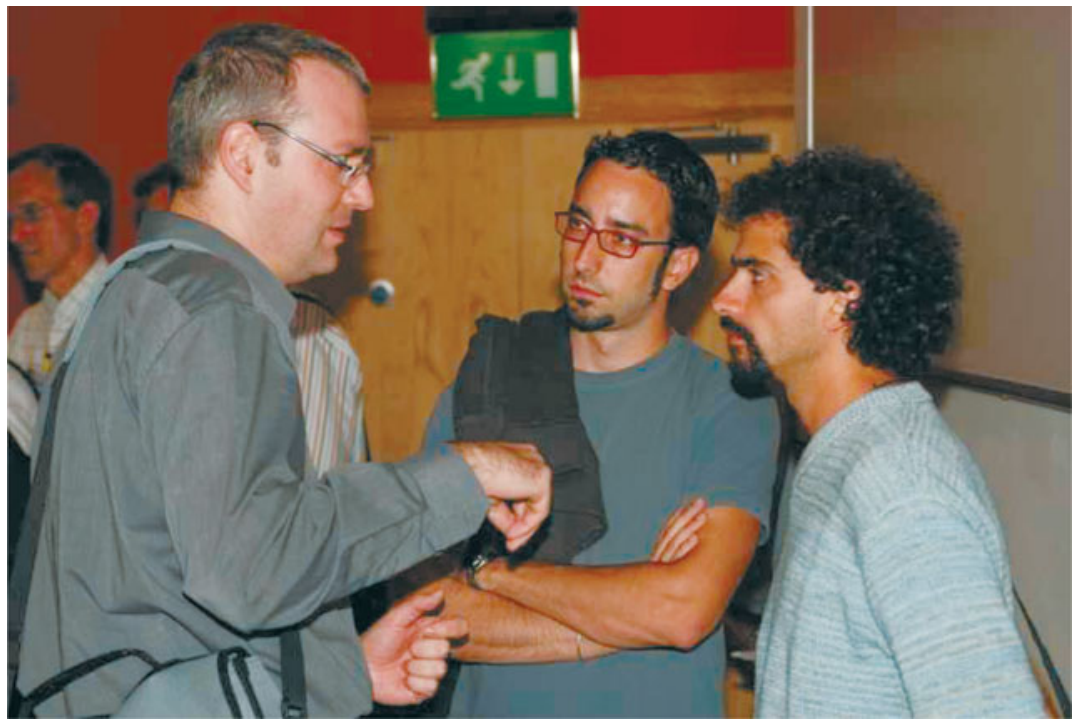

Figure 6. Enrico Maria Corsini, Jairo Méndez-Abreu and Lorenzo Morelli discussing during a coffee break. Joseph Shields is in the background on the left. 


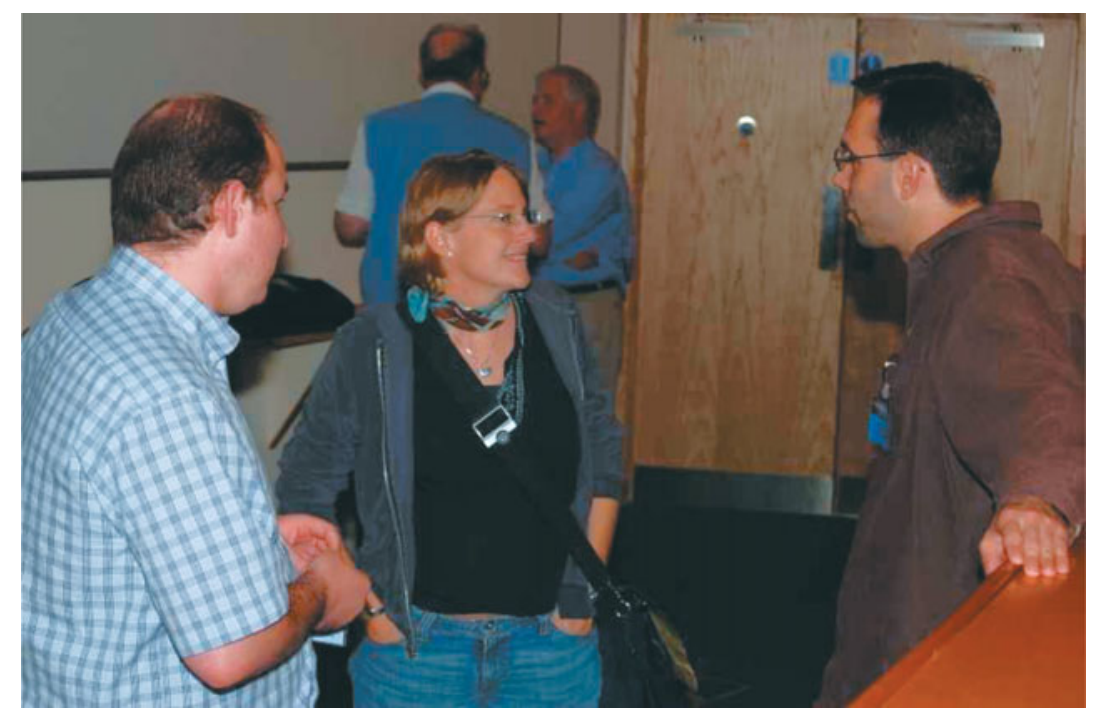

Figure 7. Jesús Falcón-Barroso, Eva Schinnerer and LOC/SOC chair Martin Bureau discussing during a coffee break. John Kormendy and Christopher O'Dea are in the background.

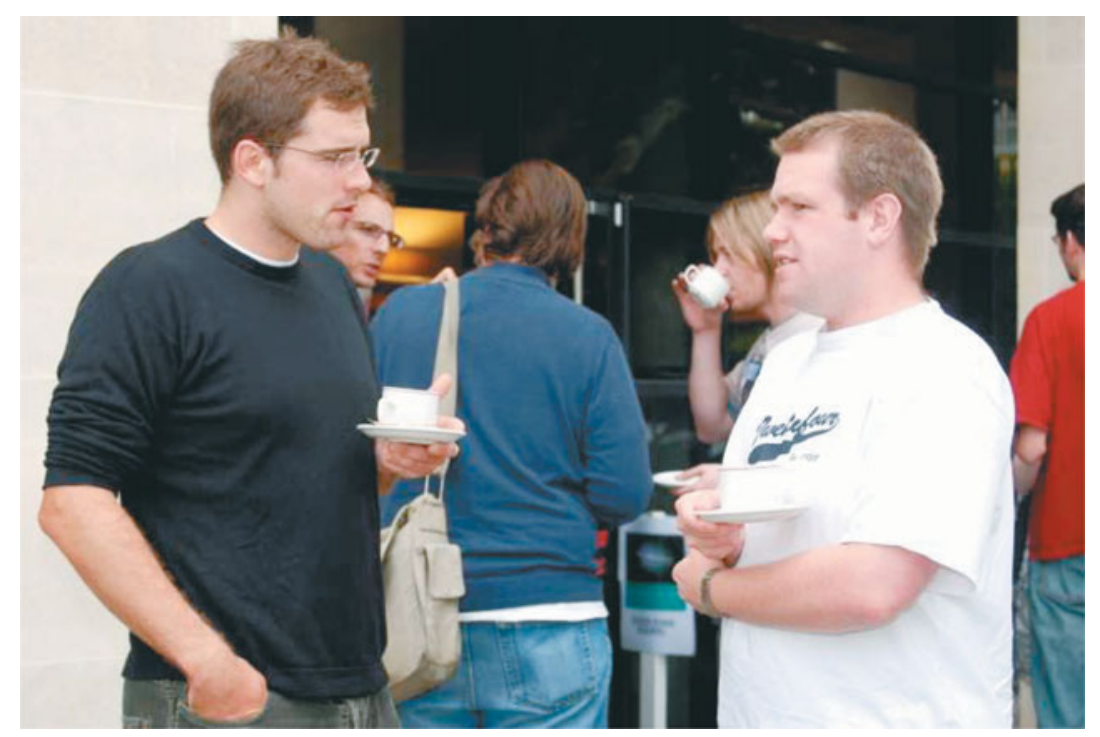

Figure 8. Ryan Houghton and Andrew Gosling discussing during a coffee break. Flavio De Lorenzi, Christian Daniel Howard, Ewan Cameron and Arjen van der Wel are visible in the background. 